\title{
Trochlear Nerve Palsy: A Review of Etiology, Incidence, Diagnostic Methods, and Treatment Alternatives
}

\author{
Ayse Gul Kocak ALTINTAS* \\ SBU Ulucanlar Eye Hospital, Turkey
}

*Corresponding author: Ayse Gul Kocak ALTINTAS, SBU Ulucanlar Eye Hospital, Turkey.

Received Date: February 24, 2020

Published Date: March 06, 2020

\begin{abstract}
s
Trochlear nerve palsy is the most common palsy among the other cranial nerve palsies. It causes superior oblique muscle palsy which presents with diplopia and the compensatory head position. Several diverse surgical alternatives are available for both congenital and acquired, superior oblique palsy. Each patient should be extensively evaluated to perform a correct operation with a high success rate. In this review etiologic factors, incidence, clinical findings and different treatment methods of superior oblique muscle palsy is presented.
\end{abstract}

\section{Introduction}

Trochlear nerve palsy (4th cranial nerve) is one of the most frequent palsies among the other cranial nerve palsy. In clinical practice, it presents with Superior oblique muscle palsy (SOP), which is the common cause of vertical and torsional strabismus. In this review etiology, incidence, diagnostic methods, and treatment alternatives with success rates of the SOP are evaluated [1-3].

\section{Incidence}

Trochlear nerve (4th cranial nerve) palsy, which innervates the superior oblique (SO) muscle, is considered to be the most common cause of cyclovertical strabismus [1-4] SOP may be congenital or acquired. Helveston et al. [2] observerd that more than $75 \%$ of the SOP cases were congenital in their series. Tollefson et al. [3] found that the annual incidence of SOP was 3.4 per 100,000 children younger than 19 years of age. But Martinez-Thompson et al. [3] reported the annual incidence of adult-onset SOP to be 6.3 cases per100,000 people in their epidemiological study. The presents of SOP in children is equal by gender but significantly higher in men than women in the adult age group [1-6].

\section{Etiology of Trochlear Never Palsy}

The most acquired trochlear nerve palsy develops secondary to trauma because the trochlear nerve is one of the most prolonged and unprotected cranial nerves. The trochlear nerve can be compromised anywhere along its long course from the dorsal midbrain to the SO muscle, mainly traversing intracranial structures, including the rigid tentorium cerebelli and cavernous sinus [1,5]. Vascular malformation, infarction such as cavernous sinus thrombosis, orbital or intracranial inflammation, infection or tumors, iatrogenic factors such as complicating orbital, sinus, or neurologic surgery cause acquired trochlear nerve or SOP [57]. The tendon of muscle may also suffer injuries such as blunt or sharp orbital traumas [1-6,7].

The leading causes of congenital trochlear nerve palsies include hypoplasia of the trochlear nerve itself or its nucleus, anatomical defects, or absence of either trochlea or the superior oblique tendon. Increased laxity, extremely elongation of SO tendon and its insertion anomalies on the sclera may mimic the trochlear nerve palsies $[1,6,7]$. 


\section{Clinical Presentation: Diplopia and Abnormal Head Position}

Clinical presentation of trochlear nerve palsy is hypertropia, due to decreased depression force and excyclotorsion, caused by paralysis of the anterior fibers of the SO muscle in the eye of the affected side. This vertical and torsional binocular misalignment causes diplopia as the main clinical symptom of SOP. To cope with vertical and torsional diplopia and regain binocular single vision, the patient adopts a compensatory abnormal head position (AHP); by this way, the eyes were placed in a particular field of gaze. The head is generally tilted towards the opposite shoulder from the hypertrophic eye, to minimize torsional diplopia due to insufficient incyclotorsion of SO, and the chin lowered to decrease the vertical deviation. In congenital SOP, the patient usually presents with an AHP; children an unable to realized diplopia because the young developing brain suppresses central perception from the effected, not aligned eye. Adults with acquired SOP complained about vertical and torsional diplopia mainly in reading position, and to compensate this double vision, an AHP was spontaneously developed [5,7-9].

Longstanding, AHP in children can cause persistent and significant contracture of the sternocleidomastoid muscle, ocular torticollis, and secondary facial asymmetry. Clinical features of congenital SOP included ipsilateral hypertropia, head tilt to opposed shoulder, and facial asymmetry $[7,8]$. The estimated prevalence of childhood torticollis is $1.3 \%$ with different etiology. SOP is the most common cause among the $22.6 \%$ of non-orthopedic causes of torticollis [9-10]. Kushner reported that incomitant cyclovertical strabismus such as SOP, was the most frequent ocular etiology $(62.7 \%)$ of torticollis [11]. Head tilt due to trochlear nerve palsy can be objectively measured by using a goniometer, arc perimeter, or surgical protractor with graded markings [12].

\section{Diagnosis of Trochlear Nerve Palsy}

Trochlear nerve palsy is present with incomitant strabismus in which the vertical deviation varies in magnitude with different gaze positions relative to the head. The Parks-Bielschowsky threestep test is the gold standard for the diagnosis of cyclovertical strabismus, especially for acute, unilateral SOP.

Parks-Bielschowsky three-step test consists of 1) ipsilateral hypertropia in central gaze 2) increased magnitude of hypertropia in contralateral than ipsilateral gaze due to unopposed activity of the palsied SO muscle's antagonist, the inferior oblique, that increases hypertropia in adduction. 3) greater hypertropia in the head is tilted towards the affected eye than the contralesional side. When the head is tilted towards the shoulder to the affected eye, deficit incyclotorsion from the palsied superior oblique compensated by hyperactivity of ipsilateral superior rectus as the other muscle has incycloduction effect. The hyperactivity of the ipsilateral superior rectus causes the increased magnitude of hypertropia also [13-15].

In the bilateral SOP, hypertropia alternates with both gaze position and head tilt, such as the right eye are hypertrophic in the contralateral side gaze, the left gaze while the left eye is hypertrophic in right gaze. In contrast, the right eye is hypertrophic on the same side the; right head tilt and left eye are hypertrophic on the left head tilt. The degree of excyclotorsion is higher in bilateral SOP than in unilateral cases [15-17].

High-resolution MRI studies of superior oblique structure and contractility demonstrate that Parks-Bielschowsky three-step test is only about $70 \%$ sensitive and $50 \%$ specific [17]. Magnetic resonance imaging (MRI) is an important diagnostic tool for both confirm the clinical diagnosis of trochlear nerve palsy and evaluating the its etiology. After extraocular nerve palsy, neurogenic atrophy occurs rapidly in corresponding denervated muscles. It has been reported by several investigations that superior oblique cross-sectional area significantly decreased after SOP developed. [18-19]. Manchandia and Demer observed that the superior oblique muscle cross-sectional area was decreased to $52 \%$ in the SOP side, comparing to the normal contralesional side [17].

MRI has demonstrated that many cases diagnosed clinically as SOP have an absence of superior oblique muscle atrophy but related to connective tissue abnormalities or abnormal shifts of muscle pulley. Ocular deviation in trochlear nerve palsy does not simply occur due to superior oblique muscle weakness but also changes in the ipsilateral antagonist inferior oblique muscle that is influenced by additional factors such as mechanical, histological, innervational changes [20-21].

If the inferior oblique muscle show hypertrophy or hypercontractility in the same eye of normal SO function, it implies by the term of Primary inferior oblique overaction (IOOA). Recent MRI studies utilizing that compartmental palsy in either anterior or posterior part of the superior oblique can occur, which can explain the different clinical findings of SOP [18,19,24-25]. Cases with congenital trochlear nerve palsy generally have a higher than the normal vertical fusion amplitude; in this way, the brain can tolerate a larger amount of vertical ocular misalignment and prevent diplopia. On the other hand, cases with acquired SOP typically present with vertical and torsional diplopia. Because the normal vertical fusion amplitude is equal or less than three prism diopters (PD) so, patients generally severely disabled by small degrees of hypertropia in acquired SOP [24-25].

The SOP is the most common cyclovertical strabismus with incomitance. However, in longstanding cases, the vertical strabismus may become more comitant, with similar magnitude in all directions of gaze, which cause heterogeneity of clinical presentation in following period in the same patient.

\section{Treatment of Strabismus Secondary to Trochlear Nerve Palsy}

SOP has a wide variety of clinical presentation if the patients are asymptomatic or have a few symptoms that easily cope with only observation without any treatment may be considered. Prismatic correction is an effective treatment to eliminate the symptom 
for patients who have a small and comitant vertical deviation. Nevertheless, the vast majority of a patient has symptomatic SOP and need surgical treatment. The main goal of surgery in SOP is to decrease the vertical deviation, elimination diplopia and correct AHP. In congenital SOP, early surgical intervention prevents persistent musculoskeletal changes occurring in the face and neck.

Several numbers of surgical treatment alternatives are available for different clinical presentations of SOP. In strabismus surgery, extraocular muscle contraction forces, or pulling directions are changing, which is influenced by the tightness of extra muscular and orbital connective tissues attachment [25-27]. Different surgical alternatives exist for the treatment of hypertropia in the SOP. The surgical plan choice depends on the amount of hypertropia in the central gaze, degree of elevation, and depression in adduction. When hypertropia combined with over elevation in adduction, one of the inferior oblique weakening procedures can be preferred to decrease the activity of the superior oblique's antagonist, the ipsilateral inferior oblique (IO) muscle.

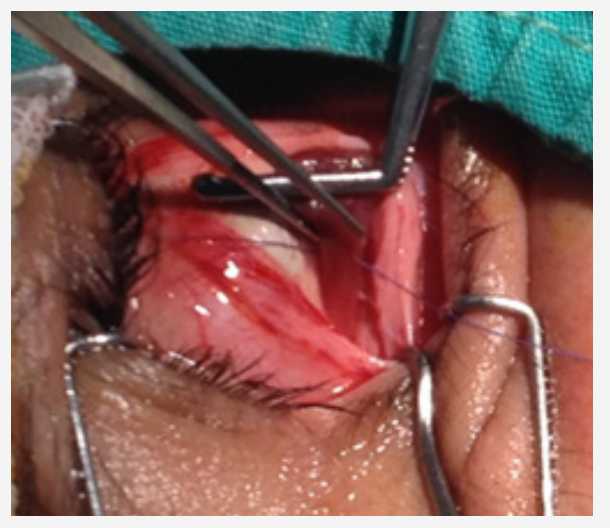

Figure 1: Disinsertion of Inferior oblique.

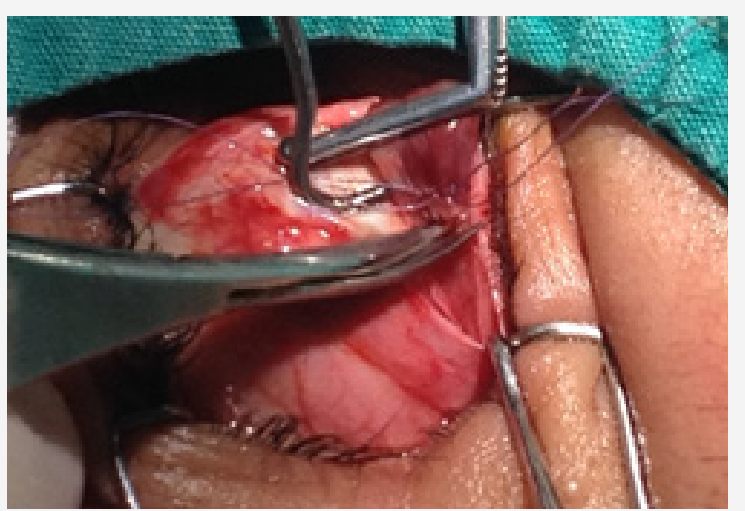

Figure 2: Excision of inferior obliue.

Several diverse IO weakening options are presented, such as recession, myectomy, total or marginal myotomy, disinsertion, anterior transposition temporal to the inferior rectus (IR) insertion, or anterior transposition nasal to IR insertion, denervation, and extirpation [28-30] (Figure 1-3).

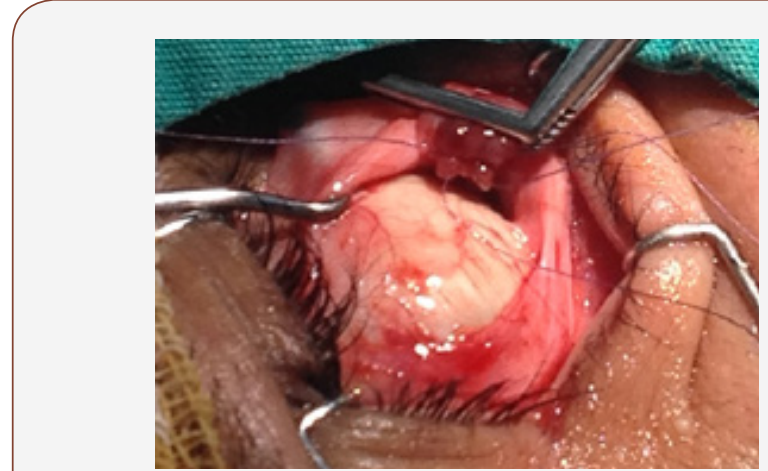

Figure 3: Recession of inferior oblique.

Parks [25] reported that the recession of the IO was the most effective and long-lasting surgery among the whole IO weakening procedures. Similar to other authors. IO recession of $8-10 \mathrm{~mm}$ corrects the 9-15 prism dioptres (PD) hypertropia in the central gaze [28-30]. Shipman et al. [29] observed an average decrease in hypertropia of 14 PD in eyes after IO myectomy, compared to 8 PD after $10 \mathrm{~mm}$ IO recession. Morad et al [30] reported the mean preoperative central gaze hypertropia of $12.5 \pm 6.2$ PD had an average correction of 9.1 PD for central gaze and 15.4 PD for contralateral gaze after $10 \mathrm{~mm}$ IOR. Metten et al. [31] reported dose-response that each $\mathrm{mm}$ of IO recession corrects $0.5^{\circ}\left(0.5^{\circ} / \mathrm{mm}\right)$ in the central position, and up to $1.4^{\circ}\left(1.4^{\circ} / \mathrm{mm}\right)$ in adduction. Yanyali [32] reported that mean reduction in hypertropia in primary gaze was 18.5 PD in eyes underwent IO anterior transposition, compared to 13. 3 PD after IO disinsertion. Clifford [33] et al reported surgical success in $82 \%$ of 96 patients after inferior oblique anterior transposition in their retrospective study over 11 years.

According to different series, IO weakening procedures have a high recurrence rate of IO over elevation. Antunes-Foschini and colleagues [34] demonstrated that extraocular muscles are histologically different from most other skeletal muscles. Extraocular muscles, maintain a process of continuous myofibriler remodeling. This remodeling is accelerated in eyes with IOOA [35]. In histological observation of Antunes-Foschini and colleagues also, documented an active modulation of individual myofibers by substantially increased numbers of activated satellite cells in the operated IO muscle, comparing the normal IO [34]. It has been shown that fixation of the IO to the orbital wall is technically challenging but effective procedure with low recurrence for weakening the action of the IO in SOP.

When the vertical deviation in central gaze severe and not combined with significant IOOA, ipsilateral superior rectus, or contralateral inferior rectus (IR) recession may be performed with or without IO weakening procedures. However, the IR recession may not effectively correct horizontal incomitance and excyclotropia $[26,36]$. In cases have torsional diplopia, from excyclotorsion, ipsilateral superior oblique tendon tucking (plication) superior oblique tendon resection, or Harada-Ito procedure, which is an 
advancement procedure the anterior portion of the superior oblique tendon may be preferred. Each of these surgeries shortens a lax SO tendon and improve the action of the superior oblique muscle [37-39]. Durnian et al. [34] reported that $71 \%$ of success rate, defined as the correction of diplopia and vertical deviation in central gaze after ipsilateral superior oblique tendon tuck, most of the authors pointed out that the amount of corrections in different operations was highly correlated with preoperative hypertropia, the greater effect of operation in the eyes with greater preoperative deviation in SOP. When the preoperative vertical deviation is lower than $15 \mathrm{PD}$, one-muscle surgeries can be sufficient, while multiple-muscle surgeries were more effective in eyes with larger deviations. Nevertheless, there is no specific guide for choosing effective surgical procedures and surgical dosage. Moreover, different surgical interventions have some specific complications rather than hypo and hypercorrection, such as iatrogenic Brown syndrome after superior oblique tuck, or anti-elevation syndrome after inferior oblique anterior transposition.

Helveston [40] reported that all of the patient developed a transient Brown syndrome in their series. At the same time, $17 \%$ of them had persistent Brown syndrome at an average of 9 months after the surgery, and they required a reoperation [14] Kaeser et al. [41] observed consecutive Brown syndrome in 18 of 20 patients after IO recession combined with SO tuck operation versus 5 of 20 patient underwent IO recession alone.

Orbital cellulitis, endophthalmitis, or retinal perforation are rarely seen but possible complications in SOP surgeries.

\section{Conclusion}

As a one of the most common cranial nerve palsy, the correct diagnosis of SOP with sensitive and specific tests is essential. Diplopia and the compensatory head position may be the first symptom similar to other neurological diseases so correct, and early treatment is essential.

Even several surgical alternatives are available for both congenital and acquired whether unilateral and bilateral, superior oblique palsy, each patient, should be extensively evaluated to perform a correct operation with a high success rate.

\section{Acknowledgement}

None.

\section{Conflict of Interest}

No conflict of interest.

\section{References}

1. Plager DA (1999) Superior oblique palsy and superior oblique myokymia. In: Rosenbaum AL, Santiago AP (Eds.), Clinical Strabismus Management. Principles and Surgical Techniques. Philadelphia: W.B. Saunders Company, USA, pp. 219-229.

2. Helveston EM, Krach D, Plager DA, Ellis FD (1992) A new classification of superior oblique palsy based on congenital variations in the tendon. Ophthalmology 99(10): 1609-1615.
3. Tollefson MM, Mohney BG, Diehl NN, Burke JP (2006) Incidence and types of childhood hypertropia: a population-based study. Ophthalmology 113(7): 1142-1145.

4. Martinez-Thompson JM, Diehl NN, Holmes JM, Mohney BG (2014) Incidence, types, and lifetime risk of adult-onset strabismus. Ophthalmology 121(4): 877-882.

5. Tamhankar MA, Biousse V, Ying GS, Prasad S, Subramanian PS, et al. (2013) Isolated third, fourth, and sixth cranial nerve palsies from presumed microvascular versus other causes: a prospective study. Ophthalmology 120(11): 2264-2269.

6. Mansour AM, Reinecke RD (1986) Central trochlear palsy. Survey of Ophthalmology 30(5): 279-97.

7. Chan TK, Demer JL (1999) Clinical features of congenital absence of the superior oblique muscle as demonstrated by orbital imaging. Journal of AAPOS 3(3):143-150.

8. Kiwak KJ (1984) Establishing an etiology for torticollis. Postgrad Med 75: 126-134.

9. Cheng JC, Au AW (1994) Infantile torticollis: A review of 624 cases. J Pediatr Orthop 14: 802-808.

10. Ballock RT, Song KM (1996) The prevalence of nonmuscular causes of torticollis in children. J Pediatr Orthop 16: 500-504.

11. Kushner BJ (1979) Ocular causes of abnormal head postures. Ophthalmology 86: 2115-2125.

12. Lau FH, Fan DS, Sun KK, Yu CB, Wong CY, et al. (2009) Residual torticollis in patients after strabismus surgery for congenital superior oblique palsy. Br J Ophthalmol 93: 1616-1619.

13. Lee JY, Kim HJ, Park KA, Oh SY, Oh SY (2018) Clinical characteristics according to the laterality of ocular torsion in unilateral superior oblique palsy. BMC Ophthalmol 18: 325-331.

14. von Noorden GK, Murray E, Wong SY (1986) Superior oblique paralysis: a review of 270 cases. Arch ophthalmol 104: 1771 -1776.

15. Kushner BJ (1989) Errors in the three-step test in the diagnosis of vertical strabismus. Ophthalmology 96: 127-132.

16. Kono R, Okanobu H, Ohtsuki H, Demer JL (2009) Absence of relationship between oblique muscle size and Bielschowsky head tilt phenomenon in clinically diagnosed superior oblique palsy. Invest Ophthalmol Vis Sci 50: $175-179$.

17. Manchandia AM, Demer JL (2014) Sensitivity of the three-step test in diagnosis of superior oblique palsy. Journal of AAPOS 18(6): 567-71.

18. Kono R, Demer JL (2003) Magnetic resonance imaging of the functional anatomy of the inferior oblique muscle in superior oblique palsy. Ophthalmology 110: 1219-1229.

19. Demer JL, Kung J, Clark RA (2011) Functional imaging of human extraocular muscles in head tilt dependent hypertropia. Invest Ophthalmol Vis Sci 52: 3023-3031.

20. Demer JL, Poukens V, Ying H, Shan X, Tian J, et al. (2010) Effects of intracranial trochlear neurectomy on the structure of the primate superior oblique muscle. Invest Ophthalmol Vis Sci 51(7): 3485-3493.

21. Clark RA, Demer JL (2011) Enhanced vertical rectus contractility by magnetic resonance imaging in superior oblique palsy. Arch Ophthalmol 129: 904-908.

22. Stager D Jr,Mc Loon Lk, Felius J (2013) Postulating a Role for Connective Tissue Elements in Inferior Oblique Muscle Overaction (An American Ophthalmological Society Thesis). Trans Am Ophthalmol Soc 111: 119-132.

23. Kushner BJ (2006) Multiple mechanisms of extraocular muscle "overaction" Arch Ophthalmol 124(5): 680-688.

24. Bharadwaj SR, Hoenig MP, Sivaramakrishnan VC, Karthikeyan B, Simonian D, et al. (2007) Variation of binocular-vertical fusion amplitude with convergence. Investigative Ophthalmology and Visual Science 48(4): $1592-1600$. 
25. Parks M. Vergences (2005) In: Tasman W, Jaeger EA (Eds.), Duane's Clinical Ophthalmology. Lippincott Williams and Wilkins.

26. Chang MY, Coleman AL, Tseng VL, Demer JL (2017) Surgical interventions for vertical strabismus in superior oblique palsy. Cochrane Database Syst Rev 11: CD012447.

27. Hendler K, Pineles SL, Demer JL, Rosenbaum AL, Velez G, et al. (2013) Does inferior oblique recession cause overcorrections in laterally incomitant small hypertropias due to superior oblique palsy? British Journal of Ophthalmology 97(1): 88-91.

28. Arici C, Oguz V (2012) The effect of surgical treatment of superior oblique muscle palsy on ocular torsion. J AAPOS 16: 21-25.

29. Shipman T, Burke J (2003) Unilateral inferior oblique muscle myectomy and recession in the treatment of inferior oblique muscle overaction: a longitudinal study. Eye 17(9): 1013-1018.

30. Morad Y, Weinstock VM, Kraft SP (2001) Outcome of inferior oblique recession with or without vertical rectus recession for unilateral superior oblique paresis. Binocul Vis Strabismus Q 16: 23-28.

31. Metten M, Link H, Staubach F, Bach M, Lagrèze WA, et al. (2008) Dose-response relationship in inferior oblique muscle recession. Graefes Arch Clin Exp Ophthalmol 246: 593-598.

32. Yanyali A, Elibol O, Talu H, Karabas L, Alp B, Caglar Y (2001) A Comparative study of the effectiveness of disinsertion and anterior transposition of the inferior oblique in the treatment of unilateral superior oblique palsy. Strabismus 9(2): 83-90.

33. Clifford L, Roos J, Dahlmann-Noor A, Vivian AJ (2015) Surgical management of superior oblique paresis using inferior oblique anterior transposition. Journal of AAPOS 19(5): 406-409.
34. Antunes-Foschini RM, Ramalho FS, Ramalho LN, Bicas HE (2006) Increased frequency of activated satellite cells in overacting inferior oblique muscles from humans. Invest Ophthalmol Vis Sci 47(8): 33603365 .

35. McLoon LK, Wirtschafter J (2003) Activated satellite cells in extraocular muscles of normal adult monkeys and humans. Invest Ophthalmol Vis Sci 44(5): 1927-1932.

36. Kaeser PF, Klainguti G, Kolling GH (2012) Inferior oblique muscle recession with and without superior oblique tendon tuck for treatment of unilateral congenital superior oblique palsy. J AAPOS 16: 26-31.

37. Durnian JM, Marsh IB (2011) Superior oblique tuck: its success as a single muscle treatment for selected cases of superior oblique palsy. Strabismus 19(4): 133-137.

38. Saunders RA (1985) Quantitated superior oblique tendon tuck in the treatment of superior oblique palsy. Am J Orthop 35: 81-89.

39. Nishimura JK, Rosenbaum AL (2002) The long-term torsion effect of the adjustable Harada-Ito procedure. J AAPOS 6(3): 141-144.

40. Helveston EM, Ellis FD (1983) Superior oblique tuck for superior oblique palsy. Aust J Ophthalmol 11: 215-220.

41. Kaeser PF, Klainguti G, Kolling GH (2012) Inferior oblique muscle recession with and without superior oblique tendon tuck for treatment of unilateral congenital superior oblique palsy. J AAPOS 16: 26-31. 